04

\title{
Объемный заряд в полимерных диэлектриках при воздействии импульсного и постоянного напряжений как фактор, влияющий на время до пробоя
}

\author{
(C) В.Ф. Важов, Е.В. Петренко \\ Инженерная школа новых производственных технологий, Томский политехнический университет, \\ Томск, Россия \\ ฯ E-mail: evgenius293@mail.ru
}

Поступила в Редакцию 11 декабря 2020 г.

В окончательной редакции 30 декабря 2020 г.

Принята к публикации 31 декабря 2020 г.

\begin{abstract}
Проведены исследования поведения объемных зарядов как во всем объеме, так и в локальных зонах рабочего объема твердых полимерных диэлектриков при длительном приложении постоянного и импульсного напряжений вплоть до образования канала пробоя. Исследования показали: чем больше величина отрицательного объемного заряда, тем меньше время до пробоя диэлектрика; появление и увеличение положительного объемного заряда как у анода, так и у катода увеличивает время жизни твердой полимерной изоляции. Предлагается использовать для контроля состояния изоляции экспериментальный метод измерения объемных зарядов в процессе эксплуатации.
\end{abstract}

Ключевые слова: высокое напряжение, полимерные диэлектрики, объемный заряд, время жизни.

DOI: 10.21883/FTT.2021.05.50811.259

\section{1. Введение}

В настоящее время не существует универсальной теории пробоя твердых диэлектриков при длительном воздействии электрического поля, когда происходит старение, деградация материала диэлектрика.

Изменение свойств полимерных диэлектриков при длительном воздействии электрического поля есть сложный комплекс механических, химических, электрических, тепловых и других процессов. Теоретическое описание этого сложного многофакторного процесса в настоящее время представляет собой неразрешимую проблему. Основным методом изучения остается экспериментальный.

Анализ литературных данных показывает, что отсутствуют систематические исследования электрической прочности твердых диэлектриков при длительном (многоимпульсном) воздействии импульсного напряжения. Чрезвычайно скудны сведения о причинах старения диэлектриков. Наиболее информативными и влияющими на процесс старения и пробоя изоляции являются протекающий ток и образование и перераспределение объемных зарядов в ее объеме [1,2].

Целью работы является исследование образования и развития объемного заряда в твердых полимерных материалах при длительном воздействии постоянного и импульсного напряжений вплоть до пробоя.

\section{2. Методика проведения экспериментов}

Концепция „слабейшего звена“, которая учитывает распределение минимальных сроков службы, на сего- дня является наиболее разработанной в теории пробоя электрической изоляции. Этому в определенной мере способствовало то, что был выведен обобщающий закон Вейбулла, который, как принято считать, является наиболее общей формой описания закономерностей старения электрической изоляции, что указывает на вероятностную форму связи времени жизни изоляции с интенсивностью воздействия внешнего электрического поля $[3,4]$. Аналитическое уравнение закона имеет вид

$$
P(E, t)=1-\exp \left(-\left(\frac{E}{E_{0}}\right)^{a}\left(\frac{t}{t_{0}}\right)^{b}\right),
$$

где $P(E, t)$ - вероятность отказа; $E, t-$ текущие значения пробивной прочности и времени до пробоя соответственно; $E_{0}, t_{0}$ - значения электрической прочности и времени до пробоя при вероятности отказа $P\left(E_{0}, t_{0}\right)=0.623 ; a, b-$ параметры формы.

Практическая ценность обобщающего закона Вейбулла заключается в возможности получения экспрессных оценок долговечности изоляции и изоляционных конструкций с любым уровнем надежности методом экстраполяции результатов ускоренных испытаний к реальным условиям эксплуатации.

Применение вероятностно-статистических методов при изучении закономерностей отказов твердых диэлектриков физически обосновано уже тем, что весь процесс формирования канала разряда с момента зарождения в объеме до полного пробоя является случайным [4].

Одним из первичных процессов, прямо или косвенно влияющих на старение и пробой твердых диэлектриков при приложении электрического поля, является образование объемного заряда (O3). Основной трудностью 

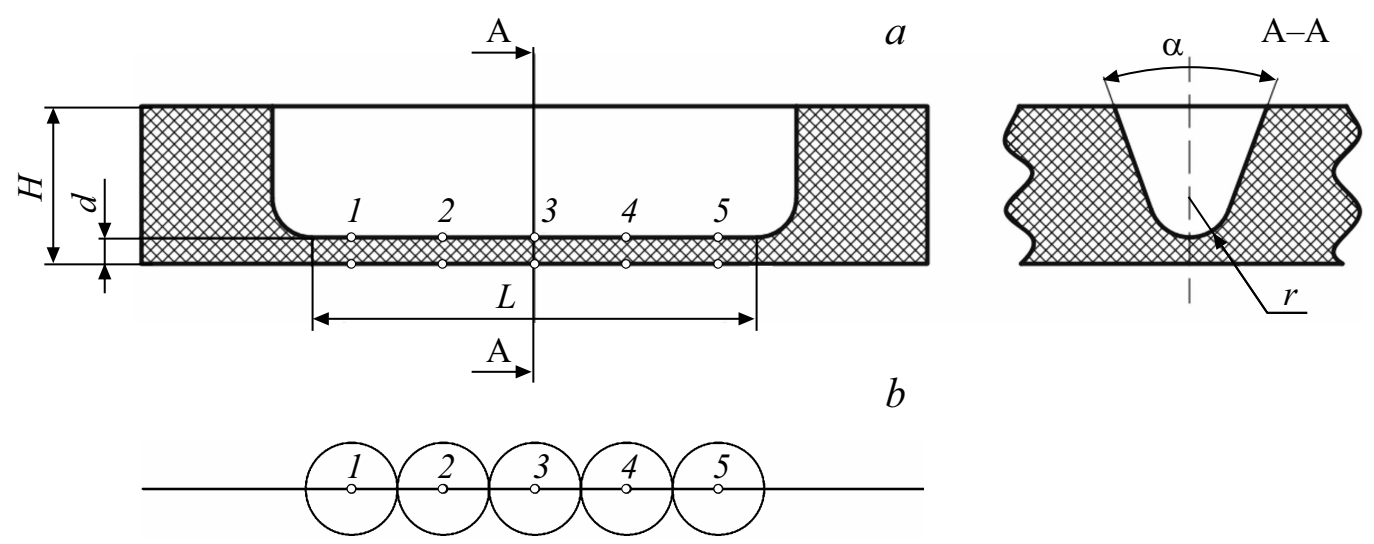

$b$

Рис. 1. Зоны измерения объемного заряда на образцах: $a-$ форма образца и расположение центров зон измерения; $b-$ зоны измерения.

прямого подтверждения обоснованности этой гипотезы является затруднение в выборе метода исследования, позволяющего экспериментально определить роль процесса образования и развития ОЗ при электрическом старении твердой изоляции вплоть до пробоя.

Для обнаружения и измерения объемных зарядов в твердых диэлектриках существует целый ряд методов: емкостной зонд, динамический конденсатор, метод теплового импульса, токи поляризации и деполяризации, метод резания, метод деполяризации пучками заряженных частиц или светом, метод импульса давления и другие. Все они обладают теми или иными недостатками. Наиболее перспективный метод волны давления (метод акустического зонда) обладает очень привлекательным свойством - он неразрушающий. Этот метод был предложен в 1976 г. во Франции [5,6]. К недостаткам этого метода можно отнести невозможность измерения локальности распределения зарядов в диэлектриках по площади электродов. Эту задачу позволяет решить альбедный $\beta$-метод, разработанный в Томском политехническом университете в 1978 г. [7]. Альбедный $\beta$-метод обладает рядом достоинств: неразрушающий, позволяет регулировать глубину зондирования, применим для непрозрачных диэлектриков, практически отсутствуют остаточные явления в измеряемом образце, особенно, при малых интенсивности и энергии зондирующего излучения, и ряд других. Этот метод относится к косвенным методам диагностики потенциалов. К недостаткам метода можно отнести большое время проведения единичных измерений и относительно низкую точность.

Изучение распределения объемных зарядов проводилось на образцах из полиэтилена низкой плотности (ПЭНП) и компаунда марки УП6-104 при длительном приложении электрического поля на постоянном и импульсном напряжениях.

В таблице приведены геометрические размеры образцов в соответствии с рис. 1.
Геометрические размеры образцов

\begin{tabular}{c|c|c|c|c|c}
\hline Материал & $d, \mathrm{~mm}$ & $r, \mathrm{~mm}$ & $L, \mathrm{~mm}$ & $\alpha$, degrees & $H, \mathrm{~mm}$ \\
\hline ПЭНП & 1.0 & 0.18 & 40 & 37 & 10 \\
УП6-104 & 1.0 & 0.23 & 40 & 39 & 10
\end{tabular}

Испытания при импульсном напряжении проводились униполярным импульсом: длительность фронта импульса $\tau_{f}=1.5 \cdot 10^{-6} \mathrm{~s}$, длительность импульса до полуспада $\tau_{u}=5 \cdot 10^{-6} \mathrm{~s}$.

На рабочие поверхности образцов напылялась платина. Высокое напряжение положительной полярности подавалось на электрод - клин. Расчет максимальной испытательной напряженности электрического поля в клинообразной системе электродов производился на основании следующего выражения:

$$
E_{t s}=E_{\max }=\frac{U_{t s}}{d} k
$$

где $U_{t s}-$ напряжение, $\mathrm{kV} ; d-$ толщина рабочего участка образца, согласно рис. $1, \mathrm{~mm} ; k-$ коэффициент неоднородности электрического поля. При этом $k$ для электродной системы клин-плоскость определялся выражением

$$
k=\frac{(0.8+0.2 \alpha)}{(r / d)^{\frac{\pi-\alpha}{2 \pi-\alpha}}},
$$

где $\alpha$ - угол, согласно рис. $1, \mathrm{rad} ; r-$ радиус дна лунки под клин в образце согласно рис. $1, \mathrm{~mm}$.

Расчет показывает, что для $d=1 \mathrm{~mm}$ коэффициент неоднородности $k=3.43$ - поле слабо неоднородное, а испытательное напряжение при $E_{t s}=50 \mathrm{kV} / \mathrm{mm}$ составляет $U_{t s}=14.5 \mathrm{kV}$. В лунку образца после напыления платины вставлялся алюминиевый конус строго по размеру, к которому подключалось испытательное напряжение.

Импульсное напряжение прикладывалось к образцам с частотой 100 pulses/s. Максимальные напряжен- 
ности электрического поля изменялись в диапазоне $E_{t s}=50-90 \mathrm{kV} / \mathrm{mm}$. Измерения О3 проводились альбедным $\beta$-методом и методом акустического зонда. Методики расчета и измерения позволяли определить напряженность электрического поля объемного заряда $E_{s c}$ для метода акустического зонда $[5,8]$ и альбедного $\beta$-метода $[7,9]$.

Образцы из ПЭНП и УП6-104 имели одинаковую форму электродов: клин - плоскость. Измерение О3 альбедным $\beta$-методом проводилось в разных зонах образца. Глубина зондирования в обоих материалах была одинакова и в среднем составляла $0.2 \mathrm{~mm}$ в областях анода и катода. На рис. 1 представлена схема зон измерения О3 на образце с протяженным электродом со стороны анода и со стороны катода. Из рис. 1 видно, что схема измерения обеспечивает контакт и даже перекрытие соседних зон измерения, что позволяет при сканировании пучком электронов контролировать всю площадь диэлектрика под электродами.

\section{3. Экспериментальные результаты}

В настоящей работе проведены исследования взаимодействия объемных зарядов и времени жизни диэлектриков при длительном воздействии электрического поля. На рис. 2 приведены распределения отказов на графике Вейбулла при длительном воздействии импульсного напряжения на образцы из полиэтилена и заливочного компаунда, для которых исследовалось влияние объемного заряда на время жизни, так же, как и при постоянном напряжении.

Из графиков видно, что все отказы можно представить отрезками прямых линий. На основании анализа подобных зависимостей сделан важный вывод, что использование распределения Вейбулла кроме прочих достоинств позволяет судить о качестве образцов в испытуемой

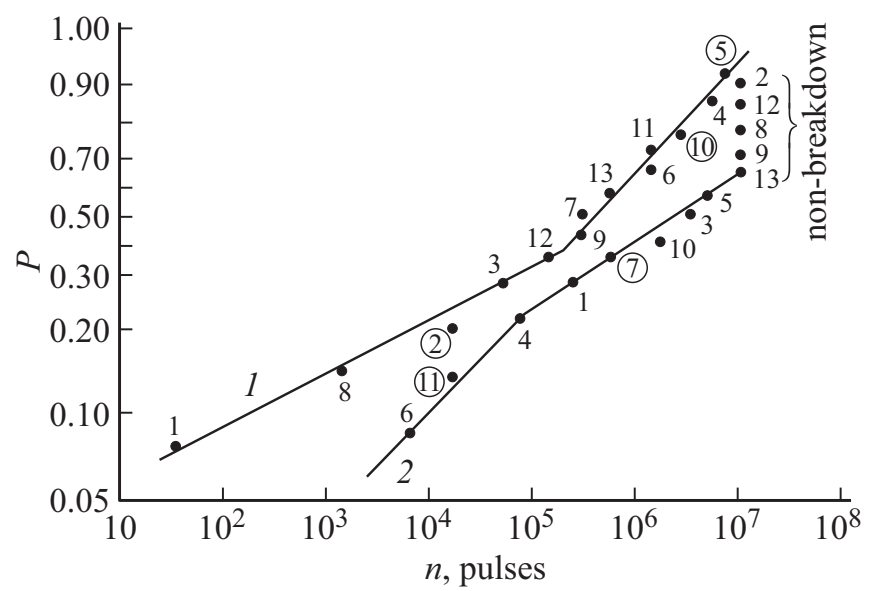

Рис. 2. Распределение отказов образцов на графике Вейбулла. 1 - ПЭНП, $2-$ УП6-104, $E_{t s}=50 \mathrm{kV} / \mathrm{mm}$; цифры у точек номера образцов в партии; отмеченные точки - образцы, в которых измерялся О3.

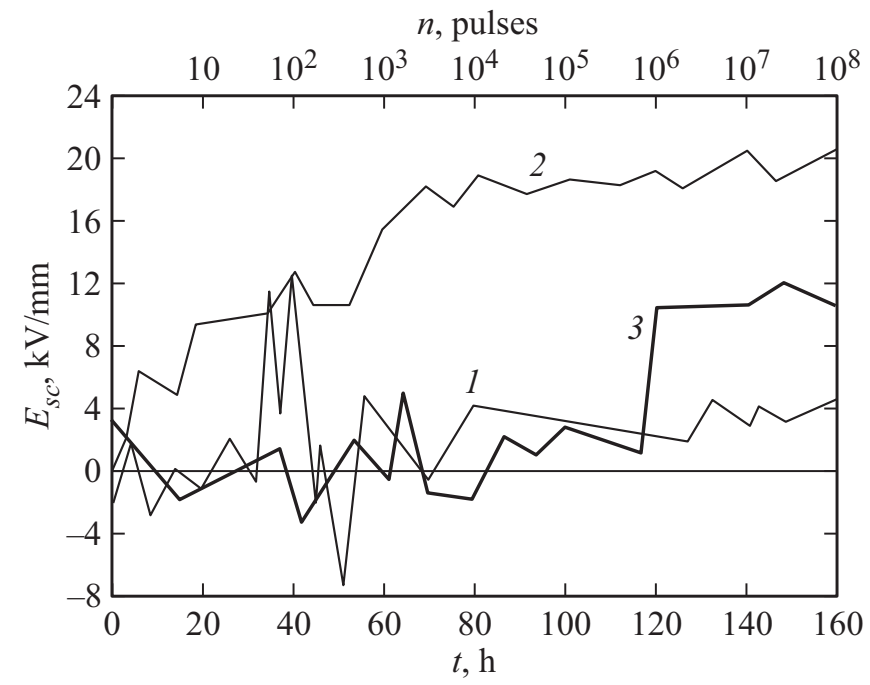

Рис. 3. Зависимость $E_{s c}$ в зоне 3 от времени $t(1,2)$ или от числа импульсов $n(3) ; 1,2$ - материал УП6-104, постоянное напряжение; 3 - материал ПЭНП, импульсное напряжение; 1 - анод; 2, 3 - катод.

партии [10]. При дальнейшем анализе результатов исследований нас постоянно будет интересовать вопрос качества испытываемых образцов. Первый участок ломаной прямой на графике Вейбулла (область ранних отказов) определяет отказы образцов с грубыми дефектами (брак). Второй и последующие участки соответствуют отказам более качественных образцов. Точка перегиба на графике Вейбулла характеризует относительное количество бракованных образцов (ось $P$ ) и число импульсов необходимое для их выявления (ось $n$ ).

Известно, что изменение максимального электрического поля, наведенного внешним электрическим полем, в объеме твердого диэлектрика носит колебательный характер [11,12]. Колебательный характер изменения $E_{s c}=f(t)$ приводит к появлению О3 разных знаков, обнаруженных нами при изменении времени испытаний образцов как вблизи анода, так и вблизи катода (рис. 3).

Накопление экспериментальных данных позволило выделить три режима в характере изменения этих колебаний - начальный, переходный, установившийся [13]. В начальном режиме изменение $E_{s c}$ имеет колебательный, хаотический, трудно предсказуемый характер как по величине, так и по знаку заряда (рис. 3).

На рис. 4 приведены значения напряженности объемного заряда $E_{s c}$ после $6 \mathrm{~h}$ испытаний в конце начального режима на постоянном напряжении во всех пяти зонах измерения в заливочном компаунде УП6-104 вблизи анода (кривая 1) и катода (кривая 2), измеренных альбедным $\beta$-методом. Видно, что изменения $E_{s c}$ носят колебательный характер, а знак заряда может быть как положительным, так и отрицательным. Длительность начального режима зависит от вида напряжения, напряженности воздействующего электрического поля, типа материала и его внутреннего состояния и т.д. В на- 


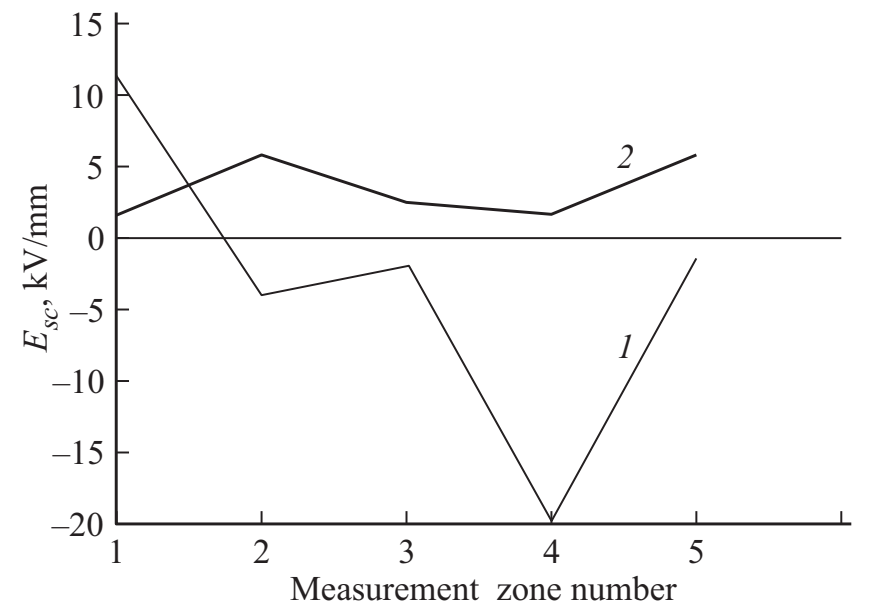

Рис. 4. Накопление $E_{s c}$ по зонам измерения после $6 \mathrm{~h}$ испытаний (начальный режим) при постоянном напряжении: 1 - анод, 2 - катод.

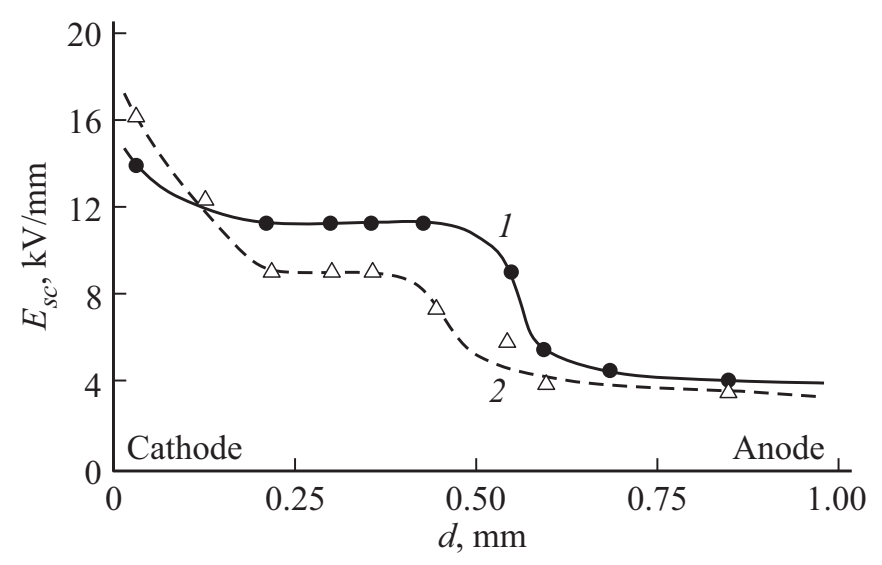

Рис. 5. Изменение напряженности электрического поля объемного заряда между анодом и катодом для ПЭНП при $E_{t s}=65 \mathrm{kV} / \mathrm{mm}: 1-n=24 \cdot 10^{6}$ pulses; $2-$ $n=102 \cdot 10^{6}$ pulses.

ших экспериментах длительность начального режима составляет: в случае постоянного напряжения - менее $8-10 \mathrm{~h}$, в случае импульсного напряжения - менее $10^{3}$ импульсов

Из рис. 3 и 4 следует, что имеет место достаточно высокая локальность накопления О3 как вблизи анода, так и катода. Локальность накопления зарядов по объему образца создает локальные электрические поля между отдельными зонами с различной величиной $E_{s c}$. Напряженности электрического поля между этими зонами могут быть значительными, что приводит к различной скорости ухудшения электроизоляционных свойств диэлектрика в разных зонах объема при длительном воздействии электрического поля. Так, в работах $[1,14]$ в ПММА обнаружены оптическими методами древовидные образования - „объемные дендриты“ по терминологии авторов работы, которые зарождаются в „бездефектных“ в исходном состоянии зонах. Очевидно, что величина и знак объемного заряда в начальном режиме зависят от исходного состояния образца и в настоящее время не предсказуемы. Увеличение времени воздействия напряжения вызывает дальнейшие изменения характера перераспределения объемного заряда. Увеличивается размах колебаний $E_{s c}$ как вблизи анода, так и вблизи катода, изменяется частота колебаний $E_{s c}$, знак заряда меняется во времени - переходный режим.

В условиях наших экспериментов длительность переходного режима составляет 60-70h при постоянном напряжении и $10^{4}-10^{5}$ импульсов при импульсном напряжении. Максимальная величина $E_{s c}$ достигает $23.5 \mathrm{kV} / \mathrm{mm}$ вблизи анода и $19.6 \mathrm{kV} / \mathrm{mm}$ вблизи катода, что превышает значения $E_{s c}$ в начальном режиме.

В переходном режиме происходит вовлечение различных механизмов генерации носителей в образование объемных зарядов. Это приводит к более интенсивному и более неравномерному перераспределению зарядов в диэлектрике, возрастанию величины $E_{s c}$. Следует отметить, что образцы с малым временем или числом импульсов до пробоя выходят из строя в переходном режиме. Но в итоге происходит стабилизация процессов перераспределения зарядов в объеме диэлектрика и переход к новому, установившемуся, режиму.

В установившемся режиме уменьшается амплитуда колебаний $E_{s c}$, колебания приобретают квазипериодический характер, знак заряда становится устойчиво положительным как у анода, так и у катода, т.е. образуется гомозаряд у анода и гетерозаряд у катода (рис. 3). Этот факт подтверждается большим количеством измерений как альбедным $\beta$-методом, так и методом акустического зонда. На рис. 5 приведено интегральное изменение напряженности поля О3 в образце ПЭНП между анодом и катодом (по толщине образца) при воздействии $24 \cdot 10^{6}$ и $102 \cdot 10^{6}$ импульсов напряжения в установившемся режиме, измеренное методом акустического зонда. Длительность фронта волны давления в данных экспериментах составляла $26 \cdot 10^{-9} \mathrm{~s}$.

Представляет практический интерес поиск взаимосвязей каких-либо характеристик О3 с временем (числом импульсов) до пробоя. Авторами для дальнейшего анализа были выбраны максимально достижимые значения $E_{s c}$ с учетом знака заряда во всех режимах изменения О3.

Для образцов с электродной системой типа клин - плоскость на рис. 6,7 приведены зависимости $E_{s c \max }=f(t, n)$ для третьей зоны измерения (рис. 1); в остальных зонах характер зависимости сохраняется. Цифры на кривых на рис. 6 соответствует номерам образцов на рис. 2 при распределении отказов в случае импульсного напряжения.

Видно, что для импульсного (рис. 6) и для постоянного (рис. 7) напряжений как для УП6-104, так и для ПЭНП зависимости имеют повторяющийся характер: в образцах, отказавших при малом времени (числе импульсов), наблюдалось отрицательное значение $E_{s c \max }$, 


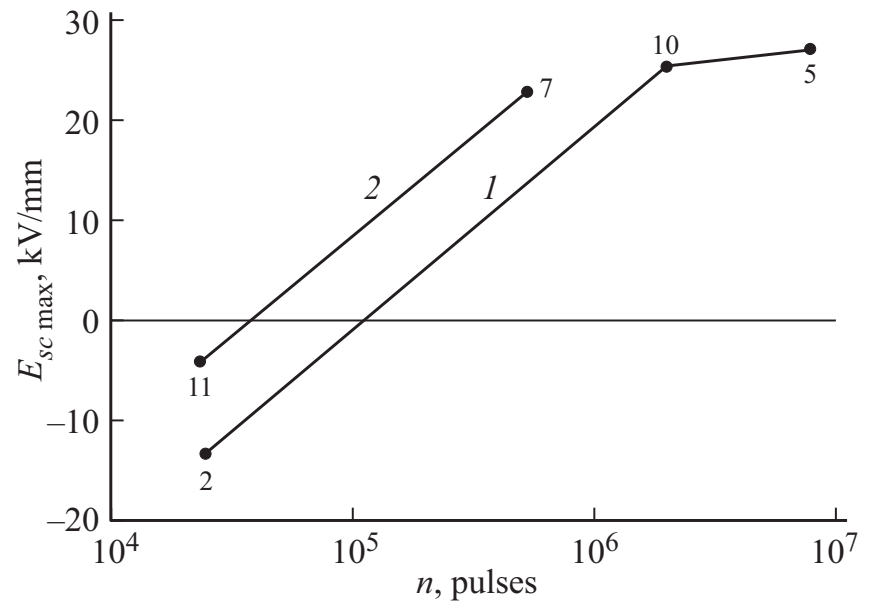

Рис. 6. Зависимость $E_{s c}$ max от числа импульсов до пробоя. 1 ПЭНП; 2 - УП6-104. Цифры у точек - номера пробитых образцов на рис. 2.

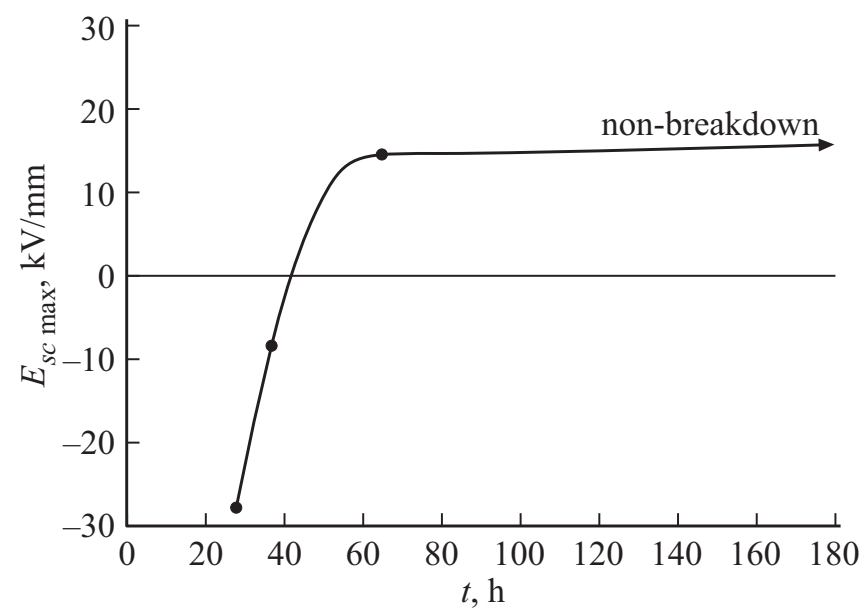

Рис. 7. Зависимость $E_{s c \max }$ от времени до пробоя для ПЭНП. Точки соответствуют пробоям образцов.

а в образцах с большим сроком службы $-E_{s c \max }$ с положительным знаком. Таким образом, уменьшение величины отрицательной $E_{s c \max }$ и увеличение положительной $E_{s c \max }$ приводит к увеличению времени (числа импульсов) до пробоя изоляции. Эти зависимости намечают пути поиска критерия оценки состояния изоляции в процессе работы и, возможно, предсказания времени до пробоя.

\section{4. Заключение}

Применение методов неразрушающего измерения объемных зарядов в твердых диэлектриках позволило исследовать их изменение в процессе длительного, вплоть до пробоя, воздействия импульсного и постоянного напряжений. Колебательный характер изменения O3 в локальных зонах диэлектрика создает области высокой напряженности электрического поля, которые могут вызвать локальный, частичный пробой в его объеме. Обнаружено, что при длительном воздействии электрического поля $\left(10^{4}-10^{5}\right.$ импульсов, 60-70 h и более) вблизи катода и анода накапливается положительный O3, т. е., гетеро- и гомозаряды соответственно, которые устойчиво сохраняются и возрастают с увеличением числа импульсов (времени) воздействия напряжения.

\section{Финансирование работы}

Исследование выполнено при финансовой поддержке РФФИ в рамках научного проекта № 19-38-90071.

\section{Конфрликт интересов}

Авторы заявляют, что у них нет конфликта интересов.

\section{Список литературы}

[1] В.Я. Ушаков, А.Л. Робежко, В.Ф. Важов, Г.В. Ефремова, В.А. Сурнин. ФТТ 5, 8, 2361 (1985).

[2] Ю.Н. Вершинин. Электронно-тепловые и детонационные процессы при электрическом пробое твердых диэлектриков. УрО РАН, Екатеринбург (2000).

[3] G. Pattini. Electrotecnica 62, 5, 433 (1975).

[4] M. Marzinotto, G. Mazzani. IEEE Electr. Insulation 31, 2, 14 (2015).

[5] P. Laurenceau, G. Dreyfus, Y. Lewiner. Phys. Rev. Lett. 38, 1, 46 (1977).

[6] P. Liu, X. Ning, Z. Peng, Z. Xiang, H. Feng, H. Zhan. IEEE Transact. Dielectrics Electrical 22, 1, 65 (2015).

[7] К.А. Дергобузов, О.Б. Евдокимов, Б.А. Кононов. Радиационная диагностика электрических потенциалов. Атомиздат, М. (1978).

[8] А.Г. Розно, В.В. Громов. Письма в ЖТФ 5, 11, 648 (1979).

[9] О.В. Андреев, О.Б. Евдокимов, Б.А. Конянов. ПТЭ 5, 227 (1978).

[10] В.Ф. Важов, С.В. Искандерова. Электричество 5, 68 (1989).

[11] К.В. Бер. Изв. АН СССР. Сер. физ. 24, 1, 36 (1960).

[12] S. Li, N. Zhao, Y. Nie, X. Wang, G. Chen, G. Teyssedre. IEEE Transact. Dielectrics Electrical 22, 1, 92 (2015).

[13] С.М. Лебедев, В.Ф. Важов, В.Я. Ушаков. ЖТФ 4, 752 (1983).

[14] M.D. Noskov, A.S. Malinoski, C.M. Cooke, K.A. Wright, A.J. Schwab. J. Appl. Phys. 92, 9, 4926 (2002).

Редактор Е.Ю. Флегонтова 